\title{
Hadronic tau jet reconstruction with particle flow algorithm at CMS
}

Lauri Wendland*†

Helsinki Institute of Physics

E-mail: lauri.wendland@cern.ch

One of the most important decay modes of the charged Higgs boson is $\mathrm{H}^{ \pm} \rightarrow \tau^{ \pm} v_{\tau}$. The strategy used to reconstruct hadronically decaying taus using particle flow reconstruction algorithm at CMS is presented. The particle flow algorithm provides a complete reconstructed event description at the level of individually reconstructed particles, which improves the tau reconstruction performance compared to previous reconstruction methods.

Prospects for Charged Higgs Discovery at Colliders

16-19 September 2008

Uppsala, Sweden

* Speaker.

${ }^{\dagger}$ on behalf of the CMS collaboration 


\section{Introduction}

If Minimal Supersymmetric Standard Model (MSSM)[1, 2, 3] describes correctly the electroweak symmetry breaking found in nature, then five Higgs bosons should exist, two of which are charged. In the maximum mixing scenario with $\mathrm{m}_{\mathrm{H}^{ \pm}}<\mathrm{m}_{\mathrm{t}}$, the dominating decay mode of the charged Higgs bosons is $\mathrm{H}^{ \pm} \rightarrow \tau^{ \pm} v_{\tau}$ [何. For $\mathrm{m}_{\mathrm{H}^{ \pm}}>\mathrm{m}_{\mathrm{t}}$, the dominating decay mode $\mathrm{H}^{ \pm} \rightarrow$ tb and the subleading decay mode $\mathrm{H}^{ \pm} \rightarrow \chi^{ \pm} \chi^{0}$ are experimentally very challenging. Thus, although the branching ratio of $\mathrm{H}^{ \pm} \rightarrow \tau^{ \pm} v_{\tau}$ is in the order of $10^{-2}$ to $10^{-3}$ for $\tan \beta=3$ and of the order of $10^{-1}$ for $\tan \beta=30$, it is experimentally the most promising decay channel for $\mathrm{m}_{\mathrm{H}^{ \pm}}>\mathrm{m}_{\mathrm{t}}$ in the maximum mixing scenario. Therefore, the $\mathrm{H}^{ \pm} \rightarrow \tau^{ \pm} v_{\tau}$ decay channel is at the moment the most important decay channel to discover the charged Higgs bosons at the LHC[5].

The $\mathrm{H}^{ \pm} \rightarrow \tau^{ \pm} v_{\tau}$ channel relies on the detection of the visible $\tau$ jet. The identification of $\tau$ jets is challenging, since it requires the processing of measurements from each subdetector: the tracking system, ECAL and HCAL calorimeters and the muon system. The $\tau$ decays may contain electrons, muons, charged hadrons, few neutral hadrons and photons. Additional difficulties are posed by secondary processes such as the decay of unstable particles, nuclear interactions with the detector material and photon conversions. In order to have an efficient and reliable $\tau$ reconstruction and identification, the particle flow algorithm has been developed to reconstruct and identify all the constituents of $\tau$ decays at particle level.

\section{Algorithm description}

The goal of the particle flow algorithm[6] is to provide a complete event description at the level of individually reconstructed particles, including non-isolated and merged particles. The complete list of particles is then in turn used to derive composite physics objects such as jet $\mathrm{E}_{\mathrm{T}}$ or missing $\mathrm{E}_{\mathrm{T}}$, which improves the performance compared to previous algorithms.

\subsection{Track reconstruction}

Since the efficiency of $\tau$ identification is directly proportional to the track reconstruction efficiency, an iterative track reconstruction algorithm is used as part of the particle flow algoritm to retain a high track reconstruction efficiency at a low fake rate. In iterative track reconstruction, very pure track seeds are used to build tracks with tight vertex cuts and a high minimum number of hits. Thus, a small fake rate is achieved at moderate tracking efficiency level. After this first step follow three iteration steps. In each iteration step the hits used in the previous step are cleaned away from the hit pool, new tracks are build from seeds deviced from the remaining hits and the track quality criteria are progressively loosened. This approach results into retaining a low fake rate and recovering a high track reconstruction efficiency. Another benefit of the iterative approach is, that the minimum reconstructable $\mathrm{p}_{\mathrm{T}}$ of the tracks is lowered to $\mathrm{p}_{\mathrm{T}}>0.3 \mathrm{GeV} / \mathrm{c}$ and the minimum reconstructable number of hits is lowered to 3 .

The iterative track reconstruction approach enables also the reconstruction of secondary processes occurring in the event. The track seeds remaining after the first iteration step are examined and the patterns are checked against signatures of nuclear interactions, photon conversions or the decay of unstable hadrons. For example, if hit density increases along a reconstructed track, a 
nuclear interaction is interpreted to have occurred. The development of the identification of these secondary processes is well underway in the particle flow algorithm.

\subsection{Identification of individual particles of the $\tau$ decay}

The strategy of identifying the different particles coming from the $\tau$ decay is to progressively associate track, ECAL and HCAL clusters to the signatures of different particle types. The associations are used to further restrict the number of different possible combinations for the so far unreconstructed particles. The matching between calorimeter and tracks is done by minimizing

$$
\chi^{2}=\left(\frac{\Delta \phi}{\sigma_{\phi}}\right)^{2}+\left(\frac{\Delta \eta}{\sigma_{\eta}}\right)^{2} .
$$

Multi-variate classifiers are used to make the association in some particle types.

The first type of associated particles are muons. Tracks, which are compatible to hits in the muon chambers, are labelled as muons and the associated objects are removed from the object pool. The ECAL and HCAL clusters on the path of muons are adjusted accordingly.

Next, tracks and ECAL clusters compatible with electron signature are associated as electrons. The energy losses of electrons are taken into account by a gaussian sum filter and the possible individual bremsstrahlung photons are labelled as photons. The final identification of electrons is based on a multi-variate classifier consisting of several variables taking into account amongst others the track and ECAL cluster compatibility and distance, track fit quality, energy loss, number of bremsstrahlung photons and the shape of the ECAL cluster. If isolated ECAL clusters are found, tracks compatible with the cluster are searched for by reconstructing tracks from the outside to the inside. Tracks compatible with these clusters are interpreted as converted photons. The final fit for the converted particle is done by making a track fit from the inside to the outside.

The next step aims to identify charged hadrons. To achieve this, calibrated HCAL clusters are compared with track momenta. If the energy of a HCAL cluster is compatible with track momentum, the objects are associated and interpreted as a charged hadron. The energy of the charged hadron is determined from a weighted average of the track $\mathrm{p}_{\mathrm{T}}$ and the calibrated energy of the HCAL cluster. If the HCAL cluster energy is incompatible with the track momentum, a neutral hadron is created from the excess in the cluster energy. Whether a neutral hadron or a photon is created is determined by the outcome of a multi-variate classifier based on the track momentum, the ratio of the ECAL and HCAL cluster energies linked to the track, compatiblity of the track to the HCAL energy cluster and the shape of the energy clusters.

Now all tracks have been associated and all remaining unassociated objects are ECAL and HCAL clusters. The remaining HCAL clusters are now assumed to be neutral hadrons and the remaining ECAL clusters are assumed to be photons.

Finally, the reconstructed particles are clustered into a jet with a cone algorithm[7] in a $(\eta, \phi)$ cone of radius 0.5 .

\section{Example of results}

Since the particle flow algorithm provides a complete description on particle level, composite physics objects may be reconstructed starting from individual particles. This approach provides 
a more natural means to calibrate composite physics object measurements. For example, since the $\tau$ decay products are mostly photons and charged pions, the particle flow based jets can take advantage of the excellent resolutions of the tracker and ECAL and calibrate the particle flow jet energy by charged particle momentum and photon energy. Additionally, the azimuthal angle of the particle flow jets is not biased by the magnetic field, since the jet azimuthal angle is reconstructed from charged particles, whose momenta has been determined at the primary vertex. Figure 1 shows the energy and azimuthal angle resolutions for particle flow based and generic calorimeter jets for $\tau$ jets with $\mathrm{p}_{\mathrm{T}}=50 \mathrm{GeV} / \mathrm{c}$. Both resolutions are observed to improve in the particle flow approach compared to generic calorimeter jets. Further improvement is expected from detailed analysis including $\pi^{0}$ 's, converted photons, etc.
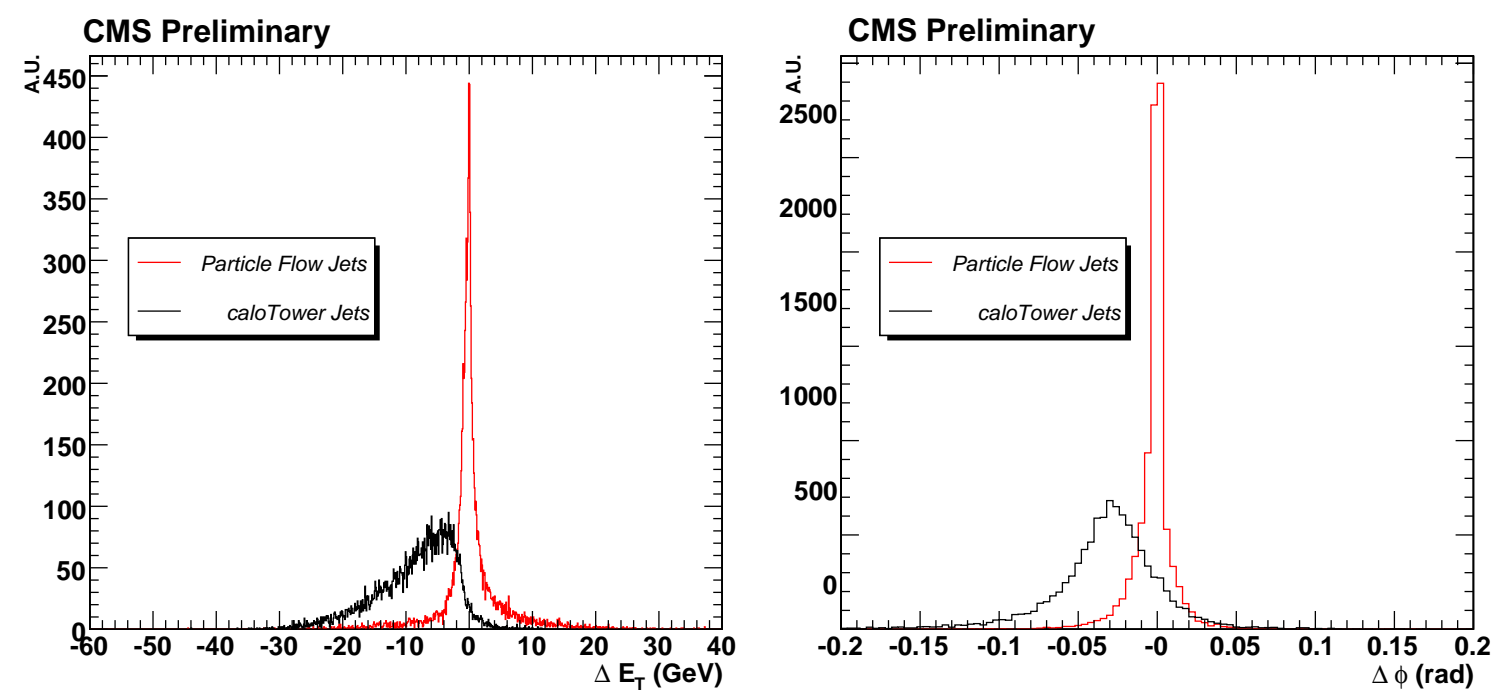

Figure 1: Comparison between particle flow reconstruction (red) and calorimeter-based (black) reconstruction of single taus with $p_{\mathrm{T}}=50 \mathrm{GeV} / c$ (typical of a $\mathrm{Z}$ decay). Left: Difference in GeV between the reconstructed and the true visible transverse momentum; Right: Difference in radian between the reconstructed and the true azimuthal angle. Jet energy corrections are not applied in either case.

It can also be shown, that the transverse missing energy resolution can be improved by a particle plow based approach (Fig. (2)[8], where pile up and underlying event are neglected. Since hadronic $\tau$ decays consist of a small number of fairly energetic and collimated particles, the particle level approach leads to a better $\mathrm{E}_{\mathrm{T}}^{\mathrm{miss}}$ resolution than generic calorimeter jets and jet corrections.

\section{Conclusions}

To discover the charged Higgs bosons, should they exist, at the LHC, good hadronic $\tau$ reconstruction is essential, since the experimentally most promising channel for the charged Higgs boson discovery, $\mathrm{H}^{ \pm} \rightarrow \tau^{ \pm} v_{\tau}$, involves $\tau$ leptons. In order to have as good $\tau$ reconstruction as possible, the particle flow algorithm has been developped. It provides a complete event description at the level of individually reconstructed particles, also in non-isolated or merged cases. The complete list of particles can further be used to derive composite physics objects and it can be shown, that this leads to improvement of tau and $\mathrm{E}_{\mathrm{T}}^{\mathrm{miss}}$ reconstruction. 


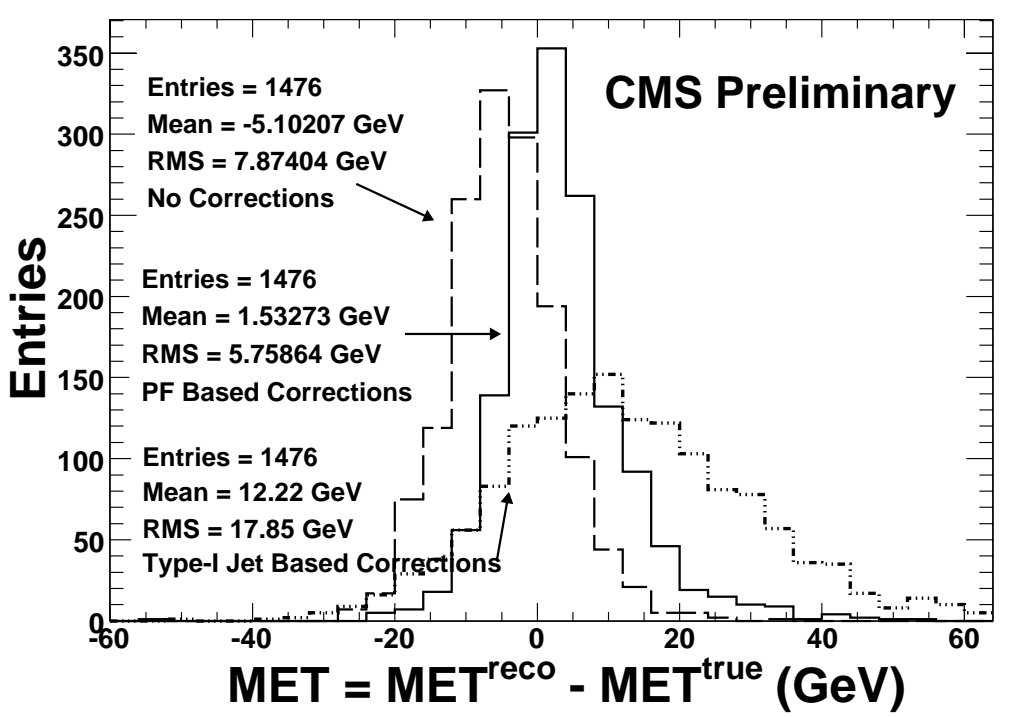

Figure 2: Distribution of $\mathrm{E}_{\mathrm{T}}^{\text {miss }}$ resolution for hadronically decaying tau leptons in $\mathrm{W}^{ \pm}$( +0 jets) $\rightarrow \tau^{ \pm} v_{\tau} \rightarrow$ hadr. events for three cases: no correction (dashed line), standard jet correction (dash-dotted line) and PFbased (solid) correction.

The implementation of the described algorithm is available for use in CMS and continuous development is ongoing to describe the existing processes in more detail and to take into account further secondary processes.

\section{References}

[1] K. Inoue, A. Kakuto, H. Komatsu and S. Takeshita, Aspects of Grand Unified Models with Softly Broken Supersymmetry, Prog. Theor. Phys. (1982) 68

[2] S. Dimopoulos and H. Georgi, Softly Broken Supersymmetry and SU(5), Nucl. Phys. B (1981) 193

[3] N. Sakai, Naturalness in Supersymmetric Guts, Zeit.Phys. C (1981) 11

[4] S. Heinemeyer, W. Hollik, P. Slavich and G. Weiglein, FeynHiggs: A program for the calculation of the masses of the neutral CP-even Higgs bosons in the MSSM, Comput. Phys. Commun. (2000) 124

[5] The CMS Collaboration, CMS Physics TDR: Volume II, Physics Performance, CERN/LHCC (2006) 2006-021

[6] The CMS Collaboration, CMS Strategies for tau reconstruction and identification using particle flow techniques, CMS PAS (2008) PFT-08-001

[7] The CMS Collaboration, Performance of Jet Algorithms in CMS, CMS PAS (2007) JME-07-003

[8] The CMS Collaboration, $\mathrm{E}_{\mathrm{T}}^{\text {miss }}$ performance in CMS, CMS PAS (2007) JME-07-001 